\title{
The Effect of Medicare Part D on Prescription Drug Spending and Health Care Use: 6 Years of Follow-up, 2007-2012
}

\author{
Taehwan Park, PhD, and Jeah Jung, PhD
}

\section{ABSTRACT}

\section{BACKGROUND: Previous studies have shown that Medicare Part D was} associated with a reduction in out-of-pocket expenditures for Medicare beneficiaries during the early years of its implementation (2006 and 2007). However, a question remains regarding the effect of Part D on out-of-pocket expenditures in the longer term.

OBJECTIVE: To evaluate the effects of Part $D$ on prescription drug expenditures and certain health care use for a longer time period using a large, nationally representative sample of Medicare beneficiaries.

METHODS: Using Medical Expenditure Panel Survey (MEPS) data from 2000 through 2005 (pre-Part D period) and from 2007 through 2012 (Part D era), this study identified a cohort of elderly Medicare beneficiaries (treatment group) and a near-elderly non-Medicare population (control group). A difference-in-differences analysis was conducted to estimate the effect of Part $D$ on prescription medication use and expenditures and outpatient visits. Propensity score weights and sampling weights were applied to obtain unbiased effect estimates accounting for complex survey designs.

RESULTS: A total of 26,585 elderly Medicare beneficiaries and 20,688 near-elderly non-Medicare beneficiaries were identified. The introduction of Part $D$ was associated with an adjusted average reduction of $\$ 105$ in annual out-of-pocket spending on prescription drugs during the post-Part $D$ period (2007 through 2012). The reduction in annual out-of-pocket spending ranged from $\$ 49$ to $\$ 152$ during the post-Part $D$ period. No significant increase was found in total prescription expenditures or prescription medication use following the introduction of Part $D$ nor were there significant changes in outpatient visits.

CONCLUSIONS: A continued reduction of Part D out-of-pocket drug expenditures was found each year from 2007 to 2012.

J Manag Care Spec Pharm. 2017;23(1):5-12

Copyright $\odot 2017$, Academy of Managed Care Pharmacy. All rights reserved.

\section{What is already known about this subject}

Prescription drug coverage through the Medicare Part D program was intended to improve access to prescription drugs by Medicare beneficiaries, with the goal of improving their health. Previous studies have shown that Part D was associated with a decrease in out-of-pocket spending on prescription drugs during the early years of its implementation.

Mixed results were reported regarding an early effect of Part D on outpatient visits.

\section{What this study adds}

This analysis found that out-of-pocket expenditures on prescription drugs decreased by $\$ 105$ annually during the 6 years (2007-2012) after the implementation of Part D. A significant year-by-year reduction in out-of-pocket expenditures indicated the persistent effect of Part D on reducing out-of-pocket drug spending.

No significant increase was found in total prescription expenditures and prescription medication use during the 6 years after the introduction of Part D.

There were no significant changes in outpatient visits after the implementation of Part D.

工 n 2003, the Medicare Prescription Drug, Improvement, and Modernization Act was enacted with the goal of improving health for seniors and other Medicare beneficiaries. One most noticeable feature of this act was the introduction of the new outpatient prescription drug benefit (Part D) program, which began on January 1, 2006. The purpose of Part D was to lower the financial hurdles that obstructed access by Medicare beneficiaries to prescription drugs by making prescription drug coverage available to beneficiaries.

Since the inception of Part D, a number of studies have evaluated the effect of Part D on out-of-pocket (OOP) drug expenditures and prescription medication use. ${ }^{1-12}$ However, many of these studies have limitations, as pointed by other investigators. ${ }^{9,13}$ For example, some early studies used claims data from pharmacy chains or single health organizations to evaluate the effect of Part D partly because nationally representative Part D claims data were not available in the early years. If the subjects in these studies were not nationally representative, the findings from the studies may have limited generalizability. ${ }^{9}$ Moreover, a majority of previous studies provided early evidence by assessing Part D's effects only up to 2007 , immediately after a transition period in 2006. There were only a few studies that examined Part D's longer-term effects beyond 2007. One study assessed Part D's effects until 2010 among oncology patients. ${ }^{12}$ Because the population used in this study was limited to patients with cancer, there is a need to investigate Part D's overall effects on access by Medicare beneficiaries to medications regardless of disease type. Other recent studies evaluated the effects of Part D on hospitalizations and 
emergency department visits until 2009 and 2012. ${ }^{15,16}$ However, these studies did not examine Part D's effects on the use of other health care, including prescription medication use and expenditures and on outpatient visits. To remedy this situation, this study evaluated the effect of Part D on prescription medication use and expenditures and outpatient visits, using a large, nationally representative sample of elderly Medicare beneficiaries. Six years of pre-Part D (2000-2005) and postPart D (2007-2012) data were used to estimate the long-term effect of Part D on spending, the use of prescription drugs, and outpatient visits.

\section{Methods}

\section{Data}

Data for this study were obtained from the Household Component file of the Medical Expenditure Panel Survey (MEPS), a nationally representative survey sponsored by the Agency for Healthcare Research \& Quality and the National Center for Health Statistics. In particular, MEPS data from Panel 4 to Panel 17 were used for the years 2000 through 2012. MEPS collects comprehensive data on health care use, expenditures, insurance coverage, and sources of payment for the civilian noninstitutionalized U.S. population. ${ }^{17}$ These data are collected using an overlapping panel design in which each panel is interviewed for a series of 5 rounds over 30 months.

\section{Study Sample}

The Part D group comprised MEPS respondents aged 65 years and older who reported that they were Medicare beneficiaries during the 2000 through 2012 survey waves (treatment group). The respondents in the 2006 wave were not included in this study because year 2006 was the transition year, thereby capturing only partial effects of Part D. To isolate the effect of the Medicare program, individuals were excluded who were concurrently enrolled in other programs such as TRICARE, Medicaid (including dual eligible), and the Veterans Health Administration (VHA). A near-elderly cohort (non-Part D group) was a control group because of their similarity with Medicare beneficiaries, following the approach in previous studies. ${ }^{4,10,12,18}$ This cohort comprised respondents who were aged 55 to 63 years and who were nonenrollees in Medicare, TRICARE, Medicaid, and the VHA during the years 20002012. As in similar studies, ${ }^{12,19}$ individuals aged 64 years were excluded because they were partially eligible for Part D; individuals aged under 55 years were also excluded to avoid variations in medication use, since women in this age group are subject to using medications for reproduction and contraception, as pointed out in similar studies..$^{10,12,19}$
After identifying the treatment group (individuals aged $\geq 65$ covered by Medicare) and the control group (near-elderly individuals not covered by Medicare), a propensity score weighting strategy was employed to control for unequal risk distribution between the 2 groups. The propensity score weights in combination with survey weights was advocated as necessary to estimate unbiased treatment effect in a survey study. ${ }^{20}$ Thus, this study used a standardized mortality/morbidity ratio (SMR)-weighted approach to estimate changes in prescription drug expenditures and health care use among the treatment group after the implementation of Part D. The SMR-weighted approach is very similar to the propensity score matching method except that the SMR-weighted approach requires no matching range and retains the entire study population..$^{21}$ In particular, this propensity score weighting method is useful when a study's goal is to generalize the final effect estimates to the original survey target population. ${ }^{22}$ Accordingly, individuals in the treatment group in this study received a weight of 1 , while those in the control group were weighted by $\hat{e} /(1-\hat{e})$, where $\hat{e}$ is the estimated propensity score. The propensity score weights were then multiplied by the survey weights to generate a new weight. The effect of Part D was estimated from a weighted regression incorporating these composite weights and the complex survey design elements. ${ }^{23}$

\section{Statistical Analysis}

This study's primary outcomes of interest were prescription medication-related expenditures (OOP expenses and total expenses on prescription drugs), prescription medication use, and outpatient visits. Specifically, OOP drug expenses were defined as a direct payment for prescription medications by the MEPS respondents; total prescription medication spending included all amounts paid by respondents and third-party payers for each prescription medication purchased; prescription medication use was measured using the number of new prescription medications and refills during the calendar year; and outpatient visits was measured by the annual number of outpatient visits. All costs were adjusted to 2012 U.S. dollars using the Consumer Price Index.

To compare the baseline characteristics between the treatment and control groups, independent t-tests for continuous variables and chi-square tests for categorical variables were conducted. The baseline characteristics were age, gender, comorbidities, race, marital status, body mass index, education, census region, poverty category, and self-reported health.

A difference-in-differences model was used to estimate the effect of Part D on each outcome. As noted earlier, the treatment group in this study included individuals aged $\geq 65$ covered by Medicare. To separate the Part D effect from changes in the outcomes by the lapse of time ("secular trends"), near-elderly individuals not covered by Medicare were used as the control group. In other words, changes in the outcomes in this control 
group during the study period captured secular or over-time trends. To compare the outcomes between these 2 groups before and after the introduction of Part D, MEPS data from 2000 through 2005 were incorporated, which represented the period before the implementation of Part D, and data from 2007 through 2012 were incorporated, representing the Part D era.

A multivariate difference-in-differences model included 2 indicator variables, one of which indicated a treatment status (coded 0 for the control group and 1 for the treatment group) and the other indicated the post-Part D period (coded 0 for the pre-Part D period and 1 for the post-Part D period), as well as the interaction between these 2 indicator variables. This interaction term was a variable of interest, capturing the effect of Part D on each outcome (i.e., how the change in each outcome between pre- and post-Part D periods in the treatment group differed from that in the control group).

To adjust for potential confounders, the model included a number of covariates, including age, gender, comorbidities, race, marital status, body mass index education, census region, poverty indicator, and self-reported health status. In particular, comorbidities were estimated by computing the Charlson Comorbidity Index, as suggested by D'Hoore et al. (1996). ${ }^{24}$

An assumption behind the difference-in-differences approach is that without the Part D introduction, the control group would have a similar trend in outcome measures to the treatment group (parallel trend assumption). To assess the validity of this parallel trend assumption, the time trend in outcomes during the pre-Part D period (2000-2005) was checked as to whether there was differential trends in outcome measures between the treatment and control groups during the pre-Part D period. Similar trends between the 2 groups would provide support for the parallel trend assumption. In addition, a year-by-year analysis was performed to explore whether the Part D effect was sustained throughout the study period. Specifically, year dummies were created, and each year dummy interacted with the indicator of the Part D period. Coefficients on these interaction terms reflected the Part D effects over years, capturing whether the immediate Part D effect shown in the previous literature was retained in relatively later years.

For analysis methods, different approaches were used depending on outcome measure. To estimate the effect of Part D on OOP and total spending on prescription drugs, a 2-part model was used, since the data included individuals with zero spending on prescription drugs. The 2-part model is considered as an optimal choice in accommodating expenditure data that are typically highly skewed to the right and zeroinflated. ${ }^{25,26}$ To analyze prescription medication use and outpatient visits (count variables), a zero-inflated negative binomial (ZINB) regression model was used because the data included those with no outpatient visits. The ZINB model was selected because it is adequate for overdispersed count outcomes with excessive zero-valued observations. ${ }^{27}$ To account for the inflated zeros, the ZINB model assumes that the distribution is a mixture of 2 distributions, that is, a logistic distribution to predict excess zeros (e.g., no use of health care) and a negative binomial distribution to model the count values (e.g., nonzero use of health care).

All analyses were conducted using STATA version 14 (StataCorp, College Station, TX) by accounting for the clustered and stratified survey design and sampling weights in the MEPS. This study was reviewed and approved by the St. Louis College of Pharmacy Institutional Review Board.

\section{Results}

The treatment group, consisting of 26,585 individuals aged 65 years or older who were covered by Medicare, served as a proxy for almost 296 million Medicare beneficiaries for 12 years (from 2000-2005 and 2007-2012), which indicates about 26 million Medicare beneficiaries per year. The control group comprised 20,688 individuals not covered by Medicare, representing over 286 million near-elderly during the same time period, which indicates about 24 million near-elderly individuals per year. Table 1 shows the characteristics of the 2 groups. The mean (standard deviation [SD]) age was $74.5(0.10)$ years for the Medicare beneficiaries and 58.8 (0.03) years for the nearelderly group. The Medicare group contained a higher percentage of women (56.9\% vs. $53.9 \%$ ) and Caucasians $(89.4 \%$ vs. $86.5 \%$ ) but a lower percentage of other race individuals than the near-elderly group (3.6\% vs. 6.1\%). In addition, Medicare beneficiaries had more comorbidities, lower body mass index, and tended to be impoverished. There were no significant differences in marital status, education, census region, and selfreported health conditions between the 2 groups.

Table 2 presents the unadjusted difference-in-difference estimates. After the introduction of Part D, the OOP expenditures for prescription medications decreased in the Medicare and near-elderly groups. The average OOP expenses decreased from $\$ 1,200$ to $\$ 623$ (a reduction of $\$ 577$ ) in the Medicare group and from $\$ 752$ to $\$ 524$ (a reduction of $\$ 228$ ) in the near-elderly group. Thus, the implementation of Part D was associated with a further reduction of \$349 in OOP medication costs for Medicare beneficiaries compared with the near-elderly group.

Increases in total prescription medication expenditures, as well as the number of prescriptions and refills after the implementation of Part D, were not significant. No significant changes were observed in outpatient visits after the introduction of Part $D(0.12$ decrease in the mean number of outpatient visits, $P=0.512$ ).

Estimates of Part D's effects obtained from the adjusted difference-in-differences regressions based on a 2-part or ZINB model are shown in Table 3. The implementation of Part D was significantly associated with a reduction in OOP expenses after controlling for the covariates (age, gender, comorbidities, race, marital status, body mass index, education, region, poverty 


\section{TABLE 1 Characteristics of the Study Sample}

\begin{tabular}{|c|c|c|c|c|c|}
\hline \multirow{2}{*}{$\begin{array}{l}\text { Characteristics } \\
\text { Age, mean }[S D]\end{array}$} & \multicolumn{2}{|c|}{ Elderly $(n=26,585)$} & \multicolumn{2}{|c|}{ Near-Elderly $(n=20,688)$} & \multirow{2}{*}{$\begin{array}{r}P \text { Value }^{\mathrm{a}} \\
<0.001\end{array}$} \\
\hline & 74.5 & {$[0.10]$} & 58.8 & {$[0.03]$} & \\
\hline \multicolumn{6}{|l|}{ Gender, \% (n) } \\
\hline Female & 56.9 & $(15,114)$ & 53.9 & $(11,147)$ & $<0.001$ \\
\hline Comorbidities, mean [SD]b & 1.9 & {$[0.04]$} & 1.1 & {$[0.05]$} & $<0.001$ \\
\hline \multicolumn{6}{|l|}{ Race, \% (n)c } \\
\hline White & 89.4 & $(23,767)$ & 86.5 & $(17,895)$ & $<0.001$ \\
\hline Black & 7.0 & $(1,861)$ & 7.4 & $(1,531)$ & 0.374 \\
\hline Others & 3.6 & $(957)$ & 6.1 & $(1,262)$ & $<0.001$ \\
\hline Married, \% (n) & 56.4 & $(14,994)$ & 57.0 & $(11,792)$ & 0.456 \\
\hline Body mass index, mean [SD] & 27.2 & {$[0.06]$} & 29.2 & {$[0.14]$} & $<0.001$ \\
\hline Education: college or postgraduate, \% (n) & 32.6 & $(8,667)$ & 33.5 & $(6,930)$ & 0.327 \\
\hline \multicolumn{6}{|l|}{ Census region, $\%(n)^{c}$} \\
\hline Northeast & 20.1 & $(5,344)$ & 20.3 & $(4,206)$ & 0.819 \\
\hline Midwest & 23.9 & $(6,354)$ & 24.8 & $(5,124)$ & 0.349 \\
\hline South & 36.1 & $(9,597)$ & 34.8 & $(7,189)$ & 0.263 \\
\hline West & 19.9 & $(5,290)$ & 20.2 & $(4,169)$ & 0.781 \\
\hline \multicolumn{6}{|l|}{ Poverty category, \% (n)c } \\
\hline Poor/negative & 7.4 & $(1,959)$ & 9.4 & $(1,945)$ & $<0.001$ \\
\hline Near poor & 6.0 & $(1,582)$ & 4.7 & $(972)$ & $<0.001$ \\
\hline Low & 18.7 & $(4,969)$ & 15.1 & $(3,124)$ & $<0.001$ \\
\hline Middle & 30.8 & $(8,180)$ & 30.8 & $(6,372)$ & 0.979 \\
\hline High & 37.2 & $(9,895)$ & 40.0 & $(8,275)$ & $<0.001$ \\
\hline \multicolumn{6}{|l|}{ Self-reported health, \% (n)c } \\
\hline Excellent & 8.0 & $(2,114)$ & 8.0 & $(1,657)$ & 0.863 \\
\hline Very good & 29.7 & $(7,904)$ & 29.1 & $(6,026)$ & 0.331 \\
\hline Good & 38.6 & $(10,256)$ & 37.2 & $(7,702)$ & 0.060 \\
\hline Fair & 19.0 & $(5,046)$ & 20.1 & $(4,158)$ & 0.165 \\
\hline Poor & 4.8 & $(1,265)$ & 5.5 & $(1,144)$ & 0.143 \\
\hline Weighted population & & 84,073 & & 37,774 & \\
\hline \multicolumn{6}{|c|}{$\begin{array}{l}\text { Independent 2-group t-tests and chi-square tests were conducted for continuous variables and categorical variables, respectively. } \\
\text { bComorbidies were estimated using the Charlson Comorbidity Index. Using ICD-9-CM codes, } 17 \text { comorbidity conditions were identifie } \\
\text { Comorbidity Index score. } \\
\text { cDifferences were tested by category using a chi-squared method, which showed the consistent findings from between-group tests above. } \\
\text { ICD-9-CM = International Classification of Diseases, Ninth Revision, Clinical Modification; SD=standard deviation. }\end{array}$} \\
\hline
\end{tabular}

indicator, and self-reported health status). By estimating an average marginal effect, an overall $\$ 105$ decrease in annual OOP spending was found on prescription drugs during the 6 years after the introduction of Part D.

Part D was not significantly associated with an increase in total spending on prescription drugs. In addition, based on the incidence rate ratio of 0.03 (95\% confidence interval $=-0.08-0.14)$ represented by the coefficients of the ZINB model, no significant increase was found in prescription medication use. The implementation of Part D did not have a significant effect on outpatient visits.

Further analyses showed significant year-by-year reductions in OOP expenditures, which indicates a sustained effect of Part D on reducing OOP spending. The yearly reductions in OOP spending ranged from $\$ 49$ to $\$ 152$ during 2007-2012 (Table 4).

\section{Discussion}

In this study, the effect of Part D on drug expenditures, prescription medication use, and outpatient visits was examined for a longer time period in elderly Medicare beneficiaries. This study revealed that the introduction of Part D was associated with a decrease in OOP prescription drug expenditures. However, total prescription expenditures, prescription medication use, and outpatient visits remained unchanged after the introduction of Part D.

A difference-in-differences approach was used for the analysis, which allowed the identification of the Part D effect after controlling for changes in the outcomes simply by the lapse of time. An adjusted reduction of $\$ 105$ was found in annual OOP spending on prescription drugs (21.8\% reduction) during the 6 years after the implementation of Part D. The mean estimate is slightly smaller compared with previous studies 
The Effect of Medicare Part D on Prescription Drug Spending and Health Care Use: 6 Years of Follow-up, 2007-2012

TABLE 2 Observed Difference-in-Differences in Outcomes of Interest

\begin{tabular}{|c|c|c|c|c|c|}
\hline Outcomes & $2000-2005$ & $2007-2012$ & Differences & DD & $P$ Value \\
\hline \multicolumn{6}{|c|}{ Out-of-pocket expenditures for prescription medications, \$ } \\
\hline Elderly & 1,200 & 623 & -577 & -349 & $<0.001$ \\
\hline Near elderly & 752 & 524 & -228 & & \\
\hline \multicolumn{6}{|c|}{ Total prescription medication expenditures, $\$$} \\
\hline Elderly & 2,036 & 2,288 & 252 & 22 & 0.897 \\
\hline Near elderly & 1,818 & 2,048 & 230 & & \\
\hline \multicolumn{6}{|c|}{ Number of prescriptions and refills } \\
\hline Elderly & 27.446 & 28.493 & 1.047 & 0.078 & 0.938 \\
\hline Near elderly & 21.782 & 22.751 & 0.969 & & \\
\hline \multicolumn{6}{|c|}{ Number of outpatient visits } \\
\hline Elderly & 1.550 & 1.107 & -0.443 & -0.116 & 0.512 \\
\hline Near elderly & 1.270 & 0.943 & -0.327 & & \\
\hline
\end{tabular}

where decreases in OOP spending were reported from $\$ 143$ to $\$ 356 .^{8-10,12}$ The larger estimates in some of the previous studies may be because those studies did not include control groups in the research design (i.e., a pre- and postperiod comparison) nor did they include those groups with zero pharmacy spending from their calculations of OOP spending, unlike this study. The results of this study, consistent with previous studies showing a decrease in OOP spending immediately after the implementation of Part D, indicated Part D's persistent effect on OOP spending on prescription drugs..$^{8-10,12}$

Slight increases (not statistically significant) were observed in total prescription expenditures and medication use after the introduction of Part D. Findings in earlier studies showed mixed results concerning this outcome. Similar to this study, several studies reported no significant changes in total prescription expenditures and prescription drug use, ${ }^{10-12}$ whereas other studies found an increase in prescription drug use ranging from $5.9 \%$ to $14.8 \% \%^{1,2,4,6,9,10}$ This study, which includes a longer follow-up period (through 2012) indicates that the introduction of Part D did not result in significant increases in prescription medication use and expenditures. The finding of an increase in prescription drug use may have been because those previous studies captured potential pent-up demand for prescription drugs. Under these circumstances, this finding suggests that this possible pent-up demand might have decreased over the years since the introduction of Part D. Indeed, the additional year-by-year analyses in this study showed that there had been an increase in prescription drug use immediately after the introduction of Part D until 2009 (an average increase of 1.53 from 2007 to 2009) but a decrease after 2009 (an average decrease of 1.34 from 2010 to 2012).

Two previous studies investigating Part D's effect on outpatient visits reached mixed conclusions. ${ }^{11,12}$ One study reported a decrease in annual outpatient visits among oncology patients, ${ }^{12}$ whereas the other study noted no significant changes in annual visits among nonelderly Medicare beneficiaries after the implementation of Part D. ${ }^{11}$ These different findings could have resulted from the heterogeneity of the study subjects. Increased access to prescription medications during the Part D era could have resulted in fewer outpatient visits by improving disease management among cancer patients but not in people with other conditions. ${ }^{12}$ The present study included only the Medicare population aged 65 years or older and found no significant effect of Part D on outpatient visits, although it was originally expected that there would be a decrease in outpatient visits after the introduction of Part $\mathrm{D}$ because of potential substitution between prescription drug use and other medical service use, as was documented in the Congressional Budget Office study. ${ }^{28}$ However, this study did not find evidence supporting a decrease in outpatient visits, which suggests that such substitution may likely occur with inpatient or emergency service use. Indeed, recent studies found that the introduction of Part D decreased hospitalization and emergency department visits for nonemergent care in the years beyond 2007. ${ }^{15,16}$

Another possible reason for this study's findings may be because prescription drugs are complements to outpatient visits for some conditions, but they might serve as substitutes for other conditions. In fact, a previous study of cancer showed that access to prescription drugs decreased outpatient visits for cancer care and management. ${ }^{12}$ Those 2 effects may have been cancelled out in the current study, which analyzed the overall effects including all conditions. Future studies could help to determine whether there is a lagged Part D effect on a decrease in outpatient visits through better management of other conditions besides cancer driven by increased access to prescription drugs.

To our knowledge, this is the first study that extended the Part D period beyond 2007 to estimate a longer-term effect of Part D on prescription drug expenditures and outpatient visits of elderly Medicare beneficiaries with no restrictions to a 
The Effect of Medicare Part D on Prescription Drug Spending and Health Care Use: 6 Years of Follow-up, 2007-2012

TABLE 3 Adjusted Difference-in-Differences Estimators of Part D Effect on Outcomes of Interest ${ }^{\mathrm{a}, \mathrm{b}}$

\begin{tabular}{l|rr|r|r}
\hline Outcomes & \multicolumn{2}{|c|}{ Coefficient (95\% CI) } & Marginal Effect & $P$ Value \\
\hline Out-of-pocket expenditures for prescription medications $^{c}$ & -0.282 & $(-0.381,-0.184)$ & -104.49 & -5.42 \\
\hline Total prescription medication expenditures $^{c}$ & 0.028 & $(-0.057,0.114)$ & 0.001 & 0.516 \\
\hline Number of prescriptions and refills $^{\mathrm{d}}$ & 0.032 & $(-0.078,0.141)$ & 0.145 & 0.570 \\
\hline Number of outpatient visits $^{\mathrm{d}}$ & -0.286 & $(-0.580,0.009)$ & -0.242 & 0.057 \\
\hline
\end{tabular}

${ }^{a}$ Covariates included in the models were age, gender, comorbidities, race, marital status, body mass index, education, region, poverty indicator, and self-reported health status.

${ }^{b}$ The estimated average propensity score (SD) for the treatment group and control group was 0.61 (0.0008) and $0.52(0.0009)$, respectively.

cResults were obtained from a 2-part model.

${ }^{d}$ Results were obtained from a zero-inflated negative binomial model.

$C I=$ confidence interval $S D=$ standard deviation

\section{TABLE 4 Adjusted Reductions in Annual Out-of-Pocket Spending During 2007-2012}

\begin{tabular}{l|c|c|c|c|c|c}
\hline Outcomes & 2007 & 2008 & 2009 & 2010 & 2011 & 2012 \\
\hline Out-of-pocket spending & $-\$ 49$ & $-\$ 98$ & $-\$ 97$ & $-\$ 107$ & $-\$ 127$ \\
& $(P=0.009)$ & $(P<0.001)$ & $(P<0.001)$ & $\begin{array}{c}-\$ 152 \\
(P<0.001)\end{array}$ \\
\hline
\end{tabular}

disease type. The pre-Part D and post-Part D periods spanned 6 years, which allowed assessment of whether Part D's effect persisted until 2012. Another strength of this study is the use of a large and nationally representative sample, which can minimize the potential limitation of studies using claims data from a single health care organization or pharmacy chain (i.e., limited generalizability if the subjects in those data were not nationally representative). In addition, this study accounted for not just survey sampling weights but also propensity score weights to account for unbalanced risk distributions between the treatment and control groups as suggested by DuGoff et al. (2014). ${ }^{20}$ This rigorous study design helped to make Part D's effect estimates robust and less biased.

\section{Limitations}

Several limitations need to be considered when interpreting the results of this study. First, as noted earlier, it was assumed that without the Part D introduction, the control group would have a similar trend in outcome measures to the treatment group (parallel trend assumption). This assumption could be violated if there was an intervention that would apply only to the control group. For example, there might have been a change in private drug coverage during the study period, which would affect only the near elderly not covered by Medicare). If the parallel trend assumption was violated, the estimates could be biased. However, the analysis of the pre-Part D period (2000-2005) showed that there were no significantly different trends in prescription drug use and expenditure between the 2 groups. While this trend might not continue in the post-Part $\mathrm{D}$ period, it suggests that any bias due to the violation of the parallel condition is likely to be small.
Second, this study focused on Part D's effect on the elderly rather than on all Medicare beneficiaries. Accordingly, this study did not include specific subpopulations such as the nonelderly disabled or beneficiaries who were dually eligible for Medicare and Medicaid. Therefore, the study results may not be generalizable to those groups. Third, all Medicare beneficiaries aged 65 years and older were included, as reported in MEPS. Therefore, Medicare beneficiaries might have been included who did not enroll in Part D, including those with no drug coverage, which account for about $10 \%$ of Medicare beneficiaries. ${ }^{29}$ Furthermore, the data did not allow for separating out beneficiaries with drug coverage before Part $\mathrm{D}$. Thus, the analysis included those with and without prescription drug coverage before Part D in the treatment. Inclusion of those with prescription drug coverage before Part D would make the estimates conservative.

Fourth, the self-reported survey may contain its own inherent limitations. For example, MEPS respondents might not recall their experiences of health care use correctly, which could lead to reporting errors and response bias. Alternatively, social desirability bias might exist if the respondents overreported the number of prescriptions and refills. However, these biases are unlikely to systematically differ between the treatment and control groups or between pre-Part D and post-Part D periods. Therefore, their effect on the findings of this study would not be substantial.

Finally, patient medication use might not be captured precisely in this study because MEPS data reflect the numbers of prescriptions filled and not the number of medications actually taken by patients. However, a previous study supported the accuracy of prescription medication use and expenditures data in the MEPS.$^{30}$ In addition, each individual's abandonment in 
the analysis could not be accounted for because this information is not available in the MEPS.

\section{Conclusions}

Medicare Part D was introduced to increase beneficiary access to prescription medications, thereby potentially improving health outcomes. Most previous studies have reported that the implementation of Part D was associated with a reduction in OOP expenditures, but they used data from an immediate follow-up period of 2006-2007. This study demonstrated that the effect of Part D in reducing OOP expenditures for prescription medications was retained up to 2012. In addition, no significant changes in total prescription expenditures and outpatient visits were observed. Although this study's findings imply that Part D is likely to have accomplished some of its intended goals, it is yet unclear whether this benefit translated to any actual improvement in health among elderly Medicare beneficiaries. Future research is warranted to assess any gains in elderly health resulting from a decrease in OOP expenditures.

\section{Authors}

TAEHWAN PARK, PhD, St. Louis College of Pharmacy, St. Louis, Missouri, and JEAH JUNG, PhD, College of Health and Human Development, The Pennsylvania State University, University Park, Pennsylvania.

AUTHOR CORRESPONDENCE: Taehwan Park, PhD,

4588 Parkview Pl., St. Louis, MO 63110. Tel.: 314.446.8193;

Fax: 314.446.8440; E-mail: Taehwan.Park@stlcop.edu.

\section{DISCLOSURES}

No funding has been received to conduct this study or prepare this manuscript. The authors have no conflicts of interest to declare.

Study concept and design were primarily contributed by Park with assistance from Jung. Both authors contributed equally to data analysis and interpretation. The manuscript was written primarily by Park, with assistance from Jung, and revised primarily by Jung.

\section{REFERENCES}

1. Lichtenberg FR, Sun SX. The impact of Medicare Part D on prescription drug use by the elderly. Health Aff (Millwood). 2007;26(6):1735-44.

2. Yin W, Basu A, Zhang JX, Rabbani A, Meltzer DO, Alexander GC. The effect of the Medicare Part D prescription benefit on drug utilization and expenditures. Ann Intern Med. 2008;148(3):169-77.

3. Chen H, Nwangwu A, Aparasu R, Essien E, Sun S, Lee K. The impact of Medicare Part D on psychotropic utilization and financial burden for community-based seniors. Psychiatr Serv. 2008;59(10):1191-97.

4. Ketcham JD, Simon KI. Medicare Part D's effects on elderly patients' drug costs and utilization. Am J Manag Care. 2008;14(11 Suppl):SP14-22.

5. Zhang Y, Donohue JM, Lave JR, O'Donnell G, Newhouse JP. The effect of Medicare Part D on drug and medical spending. N Engl J Med. 2009;361(1):52-61.
6. Joyce GF, Goldman DP, Vogt WB, Sun E, Jena AB. Medicare Part D after 2 years. Am J Manag Care. 2009;15(8):536-44.

7. Duggan M, Scott Morton F. The effect of Medicare Part D on pharmaceutical prices and utilization. Am Econ Rev. 2010;100(1):590-607.

8. Millett C, Everett CJ, Matheson EM, Bindman AB, Mainous AG. Impact of Medicare Part D on seniors' out-of-pocket expenditures on medications. Arch Intern Med. 2010;170(15):1325-30.

9. Briesacher BA, Zhao Y, Madden JM, et al. Medicare Part D and changes in prescription drug use and cost burden: national estimates for the Medicare population, 2000 to 2007. Med Care. 2011;49(9):834-41.

10. Liu FX, Alexander GC, Crawford SY, Pickard AS, Hedeker D, Walton SM The impact of Medicare Part D on out-of-pocket costs for prescription drugs, medication utilization, health resource utilization, and preference-based health utility. Health Serv Res. 2011;46(4):1104-23.

11. Nelson RE, Nelson SL, Huttner B, Gundlapalli A. The effect of Medicare Part D on health care utilization for non-elderly Medicare recipients with disabilities. Disabil Health J. 2014;7(1):64-69.

12. Kircher SM, Johansen ME, Nimeiri HS, Richardson CR, Davis MM. Impact of Medicare Part D on out-of-pocket drug costs and medical use for patients with cancer. Cancer. 2014;120(21):3378-84.

13. Polinski JM, Kilabuk E, Schneeweiss S, Brennan T, Shrank WH. Changes in drug use and out-of-pocket costs associated with Medicare Part D implementation: a systematic review. J Am Geriatr Soc. 2010;58(9):1764-79.

14. Madden JM, Graves AJ, Ross-Degnan D, Briesacher BA, Soumerai SB. Cost-related medication nonadherence after implementation of Medicare Part D, 2006-2007. JAMA. 2009;302(16):1755-56.

15. Ayyagari P, Shane DM, Wehby GL. The impact of Medicare Part D on emergency department visits. Health Econ. Published online February 11, 2016. [Epub ahead of print]

16. Kaestner R, Long C, Alexander GC. Effects of prescription drug insurance on hospitalization and mortality: evidence from Medicare Part D. National Bureau of Economic Research. September 2014. Available at: http://economics.ucr.edu/seminars_colloquia/2014-15/applied_economics/ Kaestner_2-9-15.pdf. Accessed November 16, 2016.

17. Cohen JW, Cohen SB, Banthin JS. The Medical Expenditure Panel Survey: a national information resource to support healthcare cost research and inform policy and practice. Med Care. 2009;47(7 Suppl 1):S44-50.

18. Mahmoudi E, Levy HG. How did Medicare Part D affect racial and ethnic disparities in drug coverage? J Gerontol B Psychol Sci Soc Sci. 2016;71(3):581-89.

19. Grootendorst PV, O'Brien BJ, Anderson GM. On becoming 65 in Ontario. Effects of drug plan eligibility on use of prescription medicines. Med Care. 1997;35(4):386-98.

20. Dugoff EH, Schuler M, Stuart EA. Generalizing observational study results: applying propensity score methods to complex surveys. Health Serv Res. 2014;49(1):284-303.

21. Hartzema AG, Tilson HH, Chan KA. Pharmacoepidemiology and Therapeutic Risk Management. 1st ed. Cincinnati, OH: Harvey Whitney Books Company; 2008.

22. Garrido MM, Kelley AS, Paris J, et al. Methods for constructing and assessing propensity scores. Health Serv Res. 2014;49(5):1701-20.

23. Cook BL, McGuire TG, Meara E, Zaslavsky AM. Adjusting for health status in non-linear models of health care disparities. Health Serv Outcomes Res Methodol. 2009;9(1):1-21.

24. D'Hoore W, Bouckaert A, Tilquin C. Practical considerations on the use of the Charlson Comorbidity Index with administrative data bases. J Clin Epidemiol. 1996;49(12):1429-33.

25. Olsen MK, Schafer JL. A two-part random-effects model for semicontinuous longitudinal data. Amer Statist Assoc. 2001;96(454):730-45. 
26. Manning WG, Mullahy J. Estimating log models: to transform or not to transform? J Health Econ. 2001;20(4):461-94.

27. Yau KKW, Wang K, Lee AH. Zero-inflated negative binomial mixed regression modeling of over-dispersed count data with extra zeros. Biom J. 2003;45(4):437-52.

28. Congressional Budget Office. Offsetting effects of prescription drug use on Medicare's spending for medical services. November 2012. Available at: https://www.cbo.gov/sites/default/files/cbofiles/attachments/43741-MedicalOffsets-11-29-12.pdf. Accessed November 16, 2016.
29. Kaiser Family Foundation. The Medicare prescription drug benefits. Fact sheet. September 2014. Available at: http://files.kff.org/attachment/medicareprescription-drug-benefit-fact-sheet. Accessed November 16, 2016.

30. Hill SC, Zuvekas SH, Zodet MW. Implications of the accuracy of MEPS prescription drug data for health services research. Inquiry. 2011;48(3):242-59. 